\title{
LIAR'S DOMINATION IN GRAPHS UNDER SOME OPERATIONS
}

\author{
CARLITO B. BALANDRA AND SERGIO R. CANOY, JR.
}

\begin{abstract}
A set $S \subseteq V(G)$ is a liar's dominating set (lds) of graph $G$ if $\left|N_{G}[v] \cap S\right| \geq 2$ for every $v \in V(G)$ and $\left|\left(N_{G}[u] \cup N_{G}[v]\right) \cap S\right| \geq 3$ for any two distinct vertices $u, v \in V(G)$. The liar's domination number of $G$, denoted by $\gamma_{L R}(G)$, is the smallest cardinality of a liar's dominating set of $G$. In this paper we study the concept of liar's domination in the join, corona, and lexicographic product of graphs.
\end{abstract}

\section{Introduction}

Let $G=(V(G), E(G))$ be a simple graph. The open neighborhood of $v \in V(G)$ is the set $N_{G}(v)=\{x \in V(G): x v \in E(G)\}$ and its closed neighborhood is $N_{G}[v]=N_{G}(v) \cup\{v\}$. If $S \subseteq V(G)$, then the open neighborhood of $S$ is the set $N_{G}(S)=\cup_{\nu \in S} N_{G}(v)$ and the closed neighborhood of $S$ is the set $N_{G}[S]=S \cup N_{G}(S)$ of a graph $G$. A vertex $z$ is an external private neighbor (epn) of $v \in S$ if $z \in V(G) \backslash S$ and $N(z) \cap S=\{v\}$. The set of all external private neighbors of $v$ is denoted by $e p n_{G}(v ; S)$.

A set $S \subseteq V(G)$ is a dominating set of $G$ if for every $v \in V(G) \backslash S$, there exists $u \in S$ such that $u v \in E(G)$, that is, $N_{G}[S]=V(G)$. It is a total dominating set of $G$ if $N_{G}(S)=V(G)$. $S$ is a 2-dominating set of $G$ if for every $v \in V(G) \backslash S,\left|N_{G}(v) \cap S\right| \geq 2$. A total dominating set $S$ of $G$ is a double dominating set of $G$ if it is also 2-dominating. The domination (resp. total domination, 2-domination, and double domination) number of $G$, denoted by $\gamma(G)\left(\operatorname{resp} . \gamma_{t}(G), \gamma_{2}(G)\right.$, and $\gamma_{\times 2}(G)$ ), is the smallest cardinality of a dominating (resp. total dominating, 2-dominating , and double dominating) set of $G$. Any dominating (resp. total dominating, 2-dominating, double dominating) set of $G$ of cardinality $\gamma(G)$ (resp. $\gamma_{t}(G), \gamma_{2}(G)$, and $\gamma_{\times 2}(G)$ ) is referred to as a $\gamma$-set (resp. $\gamma_{t}$-set, $\gamma_{2}$-set, and $\gamma_{\times 2}$-set) of $G$.

Received April 6, 2016, accepted November 22, 2016. 2010 Mathematics Subject Classification. 05C69.

Key words and phrases. Liar's dominating set, liar's-domination number, join, corona, lexicographic product.

Corresponding author: Carlito B. Balandra.

Research is funded by the Commission on Higher Education (CHED), Philippines under Faculty Development Program Phase II. 
A subset $S$ of $V(G)$ is called an almost dominating set of $G$ if $|V(G) \backslash N[S]| \leq 1$. The $a$ domination number of $G$, denoted by $\gamma_{a}(G)$, is the smallest cardinality of an almost dominating set of $G$. An almost dominating set of $G$ with cardinality $\gamma_{a}(G)$ is referred to as a $\gamma_{a}$-set of $G$. Since every dominating set of $G$ is an almost dominating set, it follows that $\gamma_{a}(G) \leq \gamma(G)$. Moreover, if $\gamma_{a}(G)<\gamma(G)$, then $\gamma_{a}(G)=\gamma(G)-1$. Domination in graphs as well as some of its variations can be found in [1].

In 2009, P.J. Slater et al. in [3] and [4] introduced the concept of liar's dominating set in the following way: Consider a network (modelled by a graph, say, $G$ ) where each vertex indicates a location and an intruder might be present in any of the locations in the given network. In some locations of the network are monitors or sensors which are responsible for reporting on the presence and location of the possible intruders in their respective closed neighborhoods. It is assumed that in any point of time at most one intruder can occur in the network. Further, if $S \subseteq V(G)$ is the set of monitors (or the locations of the monitors), it is assumed that at most one monitor $x \in S$ fails to report the existence of the intruder in its closed neighborhood or gives a wrong location $y$ of the intruder when the intruder is at $v$, where $y, v \in N_{G}[x]$.

Slater defined a set $S \subseteq V(G)$ to be a liar's dominating set of $G$ if for any designated vertex $v \in V(G)$ (the location of an intruder) all or all but one of the vertices in $N_{G}[v] \cap S$ report vertex $v$, and at most one vertex $w \in N_{G}[v] \cap S$ reports a vertex $y \in N_{G}[w] \backslash\{\nu\}$ or fails to report any vertex, then the vertex $v$ can be correctly identified as the designated vertex. Slater et al. in [3] characterized this concept as follows: A subset $S$ of $V(G)$ is a liar's dominating set of $G$ if and only if $\left|N_{G}[v] \cap S\right| \geq 2$ for every $v \in V(G)$ (that is, $S$ is a double dominating set of $G$ ), and $\left|\left(N_{G}[u] \cup N_{G}[v]\right) \cap S\right| \geq 3$ for any two distinct vertices $u, v \in V(G)$. The liar's domination number of $G$, denoted by $\gamma_{L R}(G)$, is the smallest cardinality of a liar's dominating set of $G$. Any liar's dominating set of $G$ with cardinality $\gamma_{L R}(G)$ is called a $\gamma_{L R}$-set of $G$. Liar's domination and its variants are also investigated by Nikodem in [2].

\section{Liar's domination in the join of graphs}

The join of two graphs $G$ and $H$ is the graph $G+H$ with vertex-set $V(G+H)=V(G) \cup V(H)$ and edge-set $E(G+H)=E(G) \cup E(H) \cup\{u v: u \in V(G), v \in V(H)\}$.

Theorem 2.1. Let $G$ be a connected graph of order $n \geq 3$ and $K_{1}=\langle\{v\}\rangle$. Then $S \subseteq V\left(K_{1}+G\right)$ is a liar's dominating set of $K_{1}+G$ if and only if one of the following holds:

(i) $S$ is a liar's dominating set of $G$;

(ii) $S=S_{1} \cup\{v\}$, where $S_{1}$ is a dominating set of $G$ such that for each $x \in S_{1}$, either $\left|\operatorname{ep} n_{G}\left(x ; S_{1}\right)\right|$ $=0$, or $\left|\operatorname{epn}_{G}\left(x ; S_{1}\right)\right|=1$ and $x \in N_{G}\left(S_{1}\right)$. 
Proof. Suppose that $S$ is an $l d s$ of $K_{1}+G$. If $v \notin S$, then, clearly, $S$ is an $l d s$ of $G$. Thus (i) holds. Suppose that $v \in S$. Then $S=S_{1} \cup\{v\}$, where $\varnothing \neq S_{1} \subseteq V(G)$. Since $S$ is a double dominating set of $K_{1}+G$, it follows that $S_{1}$ is a dominating set of $G$. Let $x \in S_{1}$. Suppose that $\left|e p n_{G}\left(x ; S_{1}\right)\right|>1$. Then there exist $y, z \in e p n_{G}\left(x ; S_{1}\right)$ such that $y \neq z$. Thus, $\mid\left(N_{G}[y] \cup N_{G}[z]\right) \cap$ $S_{1} \mid=1$. Hence, $\left|\left(N_{K_{1}+G}[y] \cup N_{K_{1}+G}[z]\right) \cap S\right|=|\{v\}|+\left|\left(N_{G}[y] \cup N_{G}[z]\right) \cap S_{1}\right|=2$. This implies that $S$ is not an $l d s$ of $K_{1}+G$, contrary to our assumption. Hence, $\left|e p n_{G}\left(x ; S_{1}\right)\right| \leq 1$. Suppose now that $\left|e p n_{G}\left(x ; S_{1}\right)\right|=1$, say $z \in e p n_{G}\left(x ; S_{1}\right)$. Since $S$ is an $l d s$ of $G+K_{1}, \mid\left(N_{K_{1}+G}[z] \cup N_{K_{1}+G}[x]\right) \cap$ $S|=1+|\left(N_{G}[z] \cup N_{G}[x]\right) \cap S_{1}|=1+1+| N_{G}(x) \cap S_{1} \mid \geq 3$. This implies that $\left|N_{G}(x) \cap S_{1}\right| \geq 1$, that is, $x \in N_{G}\left(S_{1}\right)$. This proves (ii).

For the converse, suppose first that (i) holds. Then, clearly, $S$ is an $l d s$ of $K_{1}+G$. Next, suppose that (ii) holds. Then $S=S_{1} \cup\{v\}$, where $S_{1}$ is a dominating set of $G$ satisfying the given conditions in (ii). Let $x \in V\left(K_{1}+G\right)$. If $x \in V(G) \backslash S_{1}$, then there exists $y \in S_{1} \cap N_{G}(x)$. Since $v \in N_{K_{1}+G}(x)$, it follows that $\left|N_{G+K_{1}}[x] \cap S\right|=\left|\{v\} \cup\left(N_{G}[x] \cap S_{1}\right)\right| \geq 2$. If $x=v$, then $\mid N_{K_{1}+G}[x] \cap$ $S|=|\{x\} \cup S_{1} \mid \geq 2$. If $x \in S_{1}$, then $\left|N_{K_{1}+G}[x] \cap S\right|=\left|N_{G}[x] \cap S_{1}\right|+1 \geq 2$. This shows that $S$ is a double dominating set of $K_{1}+G$.

Next, let $x, y \in V\left(K_{1}+G\right)$ such that $x \neq y$. Consider the following cases:

Case 1. $x=v$ and $y \in S_{1}$.

If $e p n_{G}\left(y ; S_{1}\right)=\varnothing$, then $\left|S_{1}\right| \geq 2$. Hence, $\left|N_{K_{1}+G}[x] \cap S\right|=1+\left|S_{1}\right| \geq 3$. This implies that $\left|\left(N_{K_{1}+G}[v] \cup N_{K_{1}+G}[y]\right) \cap S\right| \geq 3$. Suppose $\left|\operatorname{epn}_{G}\left(y ; S_{1}\right)\right|=1$. Then, by assumption, there exists $z \in S_{1} \cap N_{G}(y)$. Hence, $\left|\left(N_{K_{1}+G}[v] \cup N_{K_{1}+G}[y]\right) \cap S\right| \geq 3$.

Case 2. $x=v$ and $y \in V(G) \backslash S_{1}$.

Since $S_{1}$ is a dominating set of $G$, there exists $w \in N_{G}(y) \cap S_{1}$. If $y \notin e p n_{G}\left(w ; S_{1}\right)$, then there exists $p \in S_{1} \cap N_{G}(y)$ with $p \neq w$. Hence, $\left|S_{1}\right| \geq 2$ and $\left|\left(N_{K_{1}+G}[v] \cup N_{K_{1}+G}[y]\right) \cap S\right| \geq 3$. If $y \in \operatorname{epn}_{G}\left(w ; S_{1}\right)$, then $w \in N_{G}\left(S_{1}\right)$. Hence, $\left|S_{1}\right| \geq 2$ and $\left|\left(N_{K_{1}+G}[v] \cup N_{K_{1}+G}[y]\right) \cap S\right| \geq 3$.

Case 3. $x, y \in S_{1}$.

Then $x, y, v \in\left(N_{K_{1}+G}[x] \cup N_{K_{1}+G}[y]\right) \cap S$. Thus, $\left|\left(N_{K_{1}+G}[x] \cup N_{K_{1}+G}[y]\right) \cap S\right| \geq 3$.

Case 4. $x, y \in V(G) \backslash S_{1}$.

Since $S_{1}$ is a dominating set of $G$, there exist $z_{1}, z_{2} \in S_{1}$ such that $x z_{1}, y z_{2} \in E(G)$. If $z_{1} \neq z_{2}$, then $\left|\left(N_{G+K_{1}}[x] \cup N_{G+K_{1}}[y]\right) \cap S\right| \geq 3$ since $v \in N_{K_{1}+G}(x) \cap N_{K_{1}+G}(y)$. Suppose $z_{1}=z_{2}$. Since $\left|e p n_{G}\left(z_{1} ; S_{1}\right)\right| \leq 1$, it follows that one of $x$ and $y$, say $x$, is not in epn $\left(z_{1} ; S_{1}\right)$. Thus, $\mid\left(N_{G+K_{1}}[x] \cup\right.$ $\left.N_{G+K_{1}}[y]\right) \cap S|=1+|\left(N_{G}(x) \cup N_{G}(y)\right) \cap S_{1} \mid \geq 3$. Therefore, $S$ is an lds of $G+K_{1}$.

Case 5. $x \in S_{1}$ and $y \in V(G) \backslash S_{1}$

Since $S_{1}$ is a dominating set of $G$, there exists $z \in S_{1} \cap N_{G}(y)$. If $x \neq z$, then $\mid\left(N_{K_{1}+G}[x] \cup\right.$ $\left.N_{K_{1}+G}[y]\right) \cap S \mid \geq 3$. If $x=z$, then, by the additional property of $S_{1},\left|\left(N_{K_{1}+G}[x] \cup N_{K_{1}+G}[y]\right) \cap S\right| \geq 3$. 
Accordingly, $S$ is an lds of $K_{1}+G$.

Throughout the remaining sections we let $\Omega_{G}=\{D: D$ is a dominating set of $G$ such that for each $x \in D$, either $\left|e p n_{G}(x ; D)\right|=1$ and $x \in N_{G}(D)$ or $\left.\left|e p n_{G}(x ; D)\right|=0\right\}$, and $\gamma^{\star}(G)=\min$ $\left\{|D|: D \in \Omega_{G}\right\}$. Any set $S \in \Omega_{G}$ with $\gamma^{\star}(G)=|S|$ will be called a $\gamma^{\star}$-set of $G$.

Lemma 2.2. Let $G$ be connected graph of order $n \geq 3$. Then $\gamma^{\star}(G)+1 \leq \gamma_{L R}(G)$.

Proof. Let $D$ be a $\gamma_{L R^{-}}$set of $G$ and let $v \in D$. Since $D$ is a total dominating set of $G$, there exists $y \in D \cap N_{G}(v)$. Let $D^{\star}=D \backslash\{y\}$. Since $D$ is a 2-dominating set of $G$, it follows that $D^{\star}$ is a dominating set of $G$. Let $x \in D^{\star}$ and suppose that $\left|e p n_{G}\left(x ; D^{\star}\right)\right| \geq 2$. Let $p, q \in e p n_{G}\left(x ; D^{\star}\right)$, where $p \neq q$. If one of $p$ and $q$ is $y$, say $p=y$, then $p q \in E(G)$ since $D$ is a 2-dominating set of $G$. Hence, $\left|\left(N_{G}[p] \cup N_{G}[q]\right) \cap D\right|=2$. If $p \neq y$ and $q \neq y$, then $p y$, $q y \in E(G)$ since $D$ is a 2-dominating set of $G$. Again, this implies that $\left.\mid N_{G}[p] \cup N_{G}(q)\right) \cap D \mid=2$. Thus, in both cases, we find that $D$ is not a liar's dominating set of $G$, contrary to our assumption. Therefore $\left|e p n_{G}\left(x ; D^{\star}\right)\right| \leq 1$ for each $x \in D^{\star}$.

Next, let $x \in D^{\star}$ with $\left|e p n_{G}\left(x ; D^{\star}\right)\right|=1$, say $\{z\}=e p n_{G}\left(x ; D^{\star}\right)$. Suppose further that $x \notin N_{G}\left(D^{\star}\right)$. Then $y$ is the only neighbor of $x$ in $D$. Hence, $\left(N_{G}[z] \cup N_{G}[x]\right) \cap D=\{x, y\}$, contradicting the assumption that $D$ is an lds. Hence, $x \in N_{G}\left(D^{\star}\right)$. Since $x$ was arbitrarily chosen, it follows that $D^{\star} \in \Omega_{G}$. Consequently, $\gamma^{\star}(G) \leq\left|D^{\star}\right|=\gamma_{L R}(G)-1$.

The next result is a direct consequence of Theorem 2.1 and Lemma 2.2.

Corollary 2.3. Let $G$ be connected graph of order $n \geq 3$. Then

$$
\gamma_{L R}\left(K_{1}+G\right)=\gamma^{\star}(G)+1
$$

Example 2.4. Consider the graphs $K_{1}+G$ and $K_{1}+K_{4}$ in Figure 1 .
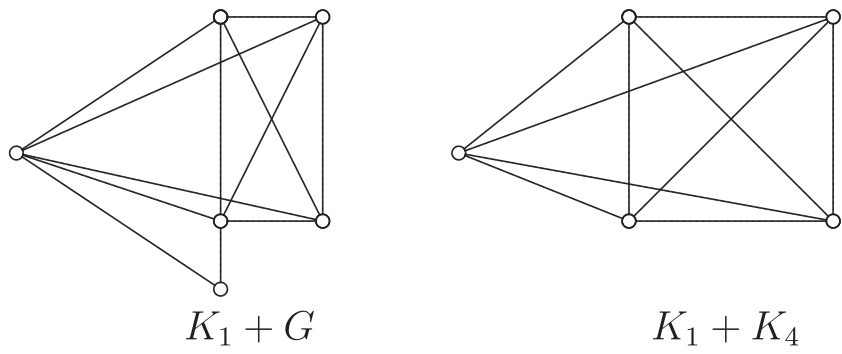

Figure 1: The graphs $K_{1}+G$ and $K_{1}+K_{4}$ 
Clearly, $1+\gamma^{\star}(G)=1+2=3<4=\gamma_{L R}(G)$. Hence, $\gamma_{L R}\left(K_{1}+G\right)=3=1+\gamma^{\star}(G)$. Also, it can easily be verified that $\gamma_{L R}\left(K_{1}+K_{4}\right)=1+\gamma^{\star}\left(K_{4}\right)=1+2=3=\gamma_{L R}\left(K_{4}\right)$.

Theorem 2.5. Let $G$ and $H$ be non-trivial connected graphs. Then $S \subseteq V(G+H)$ is a liar's dominating set of $G+H$ if and only if one of the following holds:

(i) S is a liar's dominating set of $G$.

(ii) $S$ is a liar's dominating set of $H$.

(iii) $|S \cap V(G)| \geq 3$ and $|S \cap V(H)| \geq 3$.

(iv) $S=S_{1} \cup S_{2}$, where $S_{1} \subseteq V(G)$ and $S_{2} \subseteq V(H)$ and satisfy the following:

(a) $S_{1}$ is a dominating set of $G$ such that $\left|S_{1}\right| \geq 2$ and $S_{1} \in \Omega_{G}$, and

(b) $\left|S_{2}\right|=1$, where $S_{2}$ is an almost dominating set of $H$ whenever $\left|S_{1}\right|=2$.

(v) $S=S_{1} \cup S_{2}$, where $S_{1} \subseteq V(G)$ and $S_{2} \subseteq V(H)$ and satisfy the following:

(a) $S_{2}$ is a dominating set of $H$ such that $\left|S_{2}\right| \geq 2$ and $S_{2} \in \Omega_{H}$, and

(b) $\left|S_{1}\right|=1$, where $S_{1}$ is an almost dominating set of $G$ whenever $\left|S_{2}\right|=2$.

(vi) $S=S_{1} \cup S_{2}$, where $S_{1} \subseteq V(G)$ and $S_{2} \subseteq V(H)$ and satisfy the following:

(a) $\left|S_{1}\right|=2$ and $S_{1}$ is an almost dominating set of $G$, and

(b) $\left|S_{2}\right|=2$ and $S_{2}$ is an almost dominating set of $H$.

(vii) $S=S_{1} \cup S_{2}$, where $S_{1} \subseteq V(G)$ and $\left|S_{1}\right|=2$ and $S_{2} \subseteq V(H),\left|S_{2}\right| \geq 3$ and $S_{2}$ is an almost dominating set of $H$.

(viii) $S=S_{1} \cup S_{2}$, where $S_{2} \subseteq V(H)$ and $\left|S_{2}\right|=2$ and $S_{1} \subseteq V(G),\left|S_{1}\right| \geq 3$ and $S_{1}$ is an almost dominating set of $G$.

Proof. Suppose that $S$ is an $l d s$ of $G+H$. If $S \cap V(H)=\varnothing$ or $S \cap V(G)=\varnothing$, then $S$ is an lds of $G$ or $H$. Thus, (i) or (ii) holds. Now, suppose that $S_{1}=S \cap V(G) \neq \varnothing$ and $S_{2}=S \cap V(H) \neq \varnothing$. If $\left|S_{1}\right| \geq 3$ and $\left|S_{2}\right| \geq 3$, then (iii) holds. Consider the following cases:

Case $1 .\left|S_{1}\right| \geq 2$ and $\left|S_{2}\right|=1$ or $\left|S_{1}\right|=1$ and $\left|S_{2}\right| \geq 2$.

Suppose that $\left|S_{1}\right| \geq 2$ and $\left|S_{2}\right|=1$. Since $S$ is a double dominating set of $G+H$, it follows that $S_{1}$ is a dominating set of $G$. Let $x \in S_{1}$. Suppose further that $\left|e p n_{G}\left(x ; S_{1}\right)\right| \geq 2$, say $y, z \in$ $e^{e p n_{G}}\left(x ; S_{1}\right)$, where $y \neq z$. Then $N_{G+H}[y] \cap S=N_{G+H}[z] \cap S=\{x\} \cup S_{2}$. This implies that $S$ is not a liar's dominating set of $G+H$, contrary to our assumption. Thus, $\left|\operatorname{epn}_{G}\left(x ; S_{1}\right)\right| \leq 1$. Suppose now that $\left|e p n_{G}\left(x ; S_{1}\right)\right|=1$, say $z \in e p n_{G}\left(x ; S_{1}\right)$. Since $S$ is an $l d s$ of $G+H, \mid\left(N_{G+H}[z] \cup\right.$ $\left.N_{G+H}[x]\right) \cap S|=1+|\left(N_{G}[z] \cup N_{G}[x]\right) \cap S_{1}|=1+1+| N_{G}(x) \cap S_{1} \mid \geq 3$. This implies that $\left|N_{G}(x) \cap S_{1}\right| \geq$ 1 , that is, $x \in N_{G}\left(S_{1}\right)$. Hence, $S_{1} \in \Omega_{G}$. Next, suppose that $\left|S_{1}\right|=2$ and suppose further that $\left|V(H) \backslash N_{H}\left[S_{2}\right]\right| \geq 2$. Let $a, b \in V(H) \backslash N_{H}\left[S_{2}\right]$ with $a \neq b$. Then $\left|\left(N_{G+H}[a] \cup N_{G+H}[b]\right) \cap S\right|=$ 
$\left|S_{1}\right|=2$, contrary to the assumption that $S$ is an $l d s$ of $G+H$. Thus, $S_{2}$ is an almost dominating set of $H$. This shows that (iv) holds. Similarly, (v) holds if $\left|S_{1}\right|=1$ and $\left|S_{2}\right| \geq 2$.

Case 2. $\left|S_{1}\right|=2$ and $\left|S_{2}\right|=2$.

Suppose that $\left|V(G) \backslash N\left[S_{1}\right]\right| \geq 2$. Then there exist $x, y \in V(G)$ such that $x, y \notin N\left[S_{1}\right]$. This implies that $\left|\left(N_{G+H}[x] \cup N_{G+H}[y]\right) \cap S\right|=2$. Thus, $S$ is not a liar's dominating set of $G+H$, contrary to our assumption. Hence, $\left|V(G) \backslash N\left[S_{1}\right]\right| \leq 1$. Similarly, $\left|V(H) \backslash N\left[S_{2}\right]\right| \leq 1$. Thus, (vi) holds.

Case 3. $\left|S_{1}\right|=2$ and $\left|S_{2}\right| \geq 3$ or $\left|S_{1}\right| \geq 3$ and $\left|S_{2}\right|=2$.

Suppose that $\left|S_{1}\right|=2$ and $\left|S_{2}\right| \geq 3$. Suppose further that $\left|V(H) \backslash N\left[S_{2}\right]\right| \geq 2$, say $x, y \in$ $V(H) \backslash N\left[S_{2}\right]$, where $x \neq y$. Then, $\left|\left(N_{G+H}[x] \cup N_{G+H}[y]\right) \cap S\right|=\left|S_{1}\right|=2$. This implies that $S$ is not a liar's dominating set of $G+H$, contrary to our assumption. Thus, $\left|V(H) \backslash N\left[S_{2}\right]\right| \leq 1$. This shows that (vii) holds. Similarly, (viii) holds if $\left|S_{1}\right| \geq 3$ and $\left|S_{1}\right|=2$.

The converse is clear.

It is immediate from Theorem 2.5 that $3 \leq \gamma_{L R}(G+H) \leq 6$ for any non-trivial connected graphs $G$ and $H$. The next results are also consequences of Theorem 2.5.

Corollary 2.6. Let $G$ and $H$ be non-trivial connected graphs. Then $\gamma_{L R}(G+H)=3$ if and only if at least one of the following holds:

(i) $\gamma_{L R}(G)=3$;

(ii) $\gamma_{L R}(H)=3$;

(iii) $\gamma_{a}(H)=1$ and $\gamma^{\star}(G) \leq 2$; or

(iv) $\gamma_{a}(G)=1$ and $\gamma^{\star}(H) \leq 2$.

Corollary 2.7. Let $G$ and $H$ be non-trivial connected graphs such that $\gamma_{L R}(G+H) \neq 3$. Then $\gamma_{L R}(G+H)=4$ if and only if at least one of the following holds:

(i) $\gamma_{L R}(G)=4$;

(ii) $\gamma_{L R}(H)=4$;

(iii) $\gamma_{a}(G) \leq 2$ and $\gamma_{a}(H) \leq 2$;

(iv) $\gamma^{\star}(H)=3$; or

(v) $\gamma^{\star}(G)=3$.

Corollary 2.8. Let $G$ and $H$ be non-trivial connected graphs such that $\gamma_{L R}(G+H)>4$. Then $\gamma_{p L R}(G+H)=5$ if and only if at least one of the following holds:

(i) $\gamma_{L R}(G)=5$; 
(ii) $\gamma_{L R}(H)=5$;

(iii) $\gamma_{a}(H)=3$;

(iv) $\gamma_{a}(G)=3$;

(v) $\gamma^{\star}(G)=4$; or

(vi) $\gamma^{\star}(H)=4$.

\section{Liar's domination in the corona of graphs}

The corona $G \circ H$ of two graphs $G$ and $H$ is the graph obtained by taking one copy of $G$ and $|V(G)|$ copies of $H$, and then forming the join $\langle\{v\}\rangle+H^{v}=v+H^{v}$, where $H^{v}$ is a copy of $H$, for each $v \in V(G)$.

Theorem 3.1. Let $G$ be any graph and $H$ be any non-trivial graph. Then $C \subseteq V(G \circ H)$ is a liar's dominating set of $G \circ H$ if and only if $C=A \cup\left(\cup_{v \in A} S_{v}\right) \cup\left(\cup_{u \notin A} D_{u}\right)$, where $A \subseteq V(G), S_{v} \in \Omega_{H^{v}}$ for each $v \in A$, and $D_{u}$ is a liar's dominating set of $H^{u}$ for each $u \notin A$.

Proof. Suppose that $C$ is an $l d s$ of $G \circ H$. Because $H$ is a non-trivial graph, $C \cap V\left(v+H^{\nu}\right)$ is a liar's dominating set of $v+H^{v}$ for each $v \in V(G)$. Now let $A=C \cap V(G)$. Let $v \in A$ and set $S_{v}=C \cap V\left(H^{v}\right)$. By Theorem 2.1(ii), $S_{v} \in \Omega_{H^{v}}$. Next, let $u \notin A$ and set $D_{u}=C \cap V\left(H^{u}\right)$. By Theorem 2.1(i), $D_{u}$ is a liar's dominating set of $H^{u}$.

For the converse, suppose that $C$ has the given form and the given properties. Let $z \in$ $V(G \circ H)$ and let $w \in V(G)$ such that $z \in V\left(w+H^{w}\right)$. If $w \in A$, then $E_{w}=S_{w} \cup\{w\}$ is an $l d s$ of $w+H^{w}$ by assumption and Theorem 2.1. Hence, $\left|N_{G \circ H}[z] \cap C\right| \geq\left|N_{w+H^{w}}[z] \cap E_{w}\right| \geq 2$. If $w \notin A$, then $E_{w}=D_{w}$ is an lds of $w+H^{w}$ by assumption and Theorem 2.1. Hence, $\left|N_{G \circ H}[z] \cap C\right| \geq$ $\left|N_{w+H^{w}}[z] \cap D_{w}\right| \geq 2$. Therefore, $C$ is a double dominating set of $G \circ H$.

Next, let $a, b \in V(G \circ H)$ such that $a \neq b$. Let $u, v \in V(G)$ such that $a \in V\left(u+H^{u}\right)$ and $b \in V\left(v+H^{\nu}\right)$. If $u=v$, then $\left|\left(N_{G \circ H}[a] \cup N_{G \circ H}[b]\right) \cap C\right| \geq\left|\left(N_{u+H^{u}}[a] \cup N_{u+H^{u}}[b]\right) \cap E_{u}\right| \geq 3$ since $E_{u}$ is an lds of $u+H^{u}$, where $E_{u}=S_{u} \cup\{u\}$ if $u \in A$ and $E_{u}=D_{u}$ if $u \notin A$. If $u \neq v$, then $\left|\left(N_{G \circ H}[a] \cup N_{G \circ H}[b]\right) \cap C\right| \geq\left|N_{u+H^{u}}[a] \cup E_{u}\right|+\left|N_{\nu+H^{v}}[b] \cap E_{v}\right| \geq 2+2=4$ since $E_{u}$ and $E_{v}$ are double dominating sets of $u+H^{u}$ and $v+H^{v}$, respectively, where $E_{u}=S_{u} \cup\{u\}$ if $u \in A$ and $E_{u}=D_{u}$ if $u \notin A$, and $E_{v}=S_{v} \cup\{v\}$ if $v \in A$ and $E_{v}=D_{v}$ if $v \notin A$. Therefore, $C$ is an $l d s$ of $G \circ H$.

Corollary 3.2. Let $G$ be any graph and $H$ be any non-trivial graph. Then $\gamma_{L R}(G \circ H)=|V(G)|\left(\gamma^{\star}(H)+1\right)$.

Proof. Let $S$ be a $\gamma^{\star}$-set of $H$. For each $v \in V(G)$, let $S_{\nu} \subseteq V\left(H^{\nu}\right)$ such that $\left\langle S_{\nu}\right\rangle \cong\langle S\rangle$. By Theorem 3.1, $C=V(G) \cup\left(\cup_{v \in V(G)} S_{\nu}\right)$ is an lds of $G \circ H$. Hence, $\gamma_{L R}(G \circ H) \leq|C|=|V(G)|\left(\gamma^{\star}(H)+1\right)$. 
Next, suppose that $C^{\prime}$ is a $\gamma_{L R}$-set of $G \circ H$. Then $C^{\prime}=A \cup\left(\cup_{v \in A} S_{v}\right) \cup\left(\cup_{u \notin A} D_{u}\right)$, where $A \subseteq V(G)$ and the sets $S_{\nu}$ 's and $D_{u}$ 's satisfy the properties given in Theorem 3.1. Thus, $\gamma_{L R}(G \circ$ $H)=\left|C^{\prime}\right|=|A|+\sum_{\nu \in A}\left|S_{\nu}\right|+\sum_{\nu \notin A}\left|D_{u}\right| \geq|A|+|A| \gamma^{\star}(H)+(|V(G)|-|A|) \gamma_{L R}(H)$. By Lemma 2.2, it follows that $\gamma_{L R}(G \circ H) \geq|V(G)|\left(\gamma^{\star}(H)+1\right)$. This proves the desired equality.

\section{Liar's domination in the lexicographic product of graphs}

The lexicographic product of two graphs $G$ and $H$ is the graph $G[H]$ with vertexset $V(G[H])=V(G) \times V(H)$ and edge-set $E(G[H])$ satisfying the following conditions: $\left(u_{1}, u_{2}\right)\left(v_{1}, v_{2}\right) \in E(G[H])$ if and only if either $u_{1} v_{1} \in E(G)$ or $u_{1}=v_{1}$ and $u_{2} v_{2} \in E(H)$.

Observe that a non-empty subset $C$ of $V(G[H])=V(G) \times V(H)$ can be written as $C=$ $\bigcup_{x \in S}\left(\{x\} \times T_{x}\right)$, where $S \subseteq V(G)$ and $T_{x} \subseteq V(H)$ for every $x \in S$. Henceforth, we shall use this form to denote any non-empty subset $C$ of $V(G[H])$.

Theorem 4.1. Let $G$ and $H$ be connected graphs of orders $n \geq 2$ and $m \geq 3$, respectively. A nonempty subset $C=\bigcup_{x \in S}\left(\{x\} \times T_{x}\right)$ of $V(G[H])$, where $S \subseteq V(G)$ and $\varnothing \neq T_{x} \subseteq V(H)$ for each $x \in S$, is a liar's dominating set of $G[H]$ if and only if $S$ is a dominating set of $G$ and satisfies each of the following:

(i) $T_{x}$ is a liar's dominating set of $H$ for each $x \in S \backslash N_{G}(S)$;

(ii) for each $x \in S \cap N_{G}(S)$ such that $N_{G}(x) \cap S=\{y\}$, one of the following holds:

(a) $\left|T_{y}\right| \geq 3$;

(b) $T_{x}$ is an almost dominating set and $\left|T_{y}\right|=2$;

(c) $\left|T_{y}\right|=1$ and $T_{x}$ is a dominating set such that $T_{x} \in \Omega_{H}$;

(iii) $T_{x}$ is an almost dominating set of $H$ for each $x \in S \cap N_{G}(S)$ such that $\left|N_{G}(x) \cap S\right|=2$ and $\left|T_{y}\right|=\left|T_{z}\right|=1$, where $N_{G}(x) \cap S=\{y, z\} ;$ and

(iv) for each $x \in V(G) \backslash S$,

(a) $\left|T_{y}\right| \geq 3$ whenever $N_{G}(x) \cap S=\{y\}$; and

(b) $\left|T_{y}\right| \geq 2$ or $\left|T_{z}\right| \geq 2$ whenever $N_{G}(x) \cap S=\{y, z\}$.

Proof. Suppose that $C$ is an lds of $G[H]$. Since $C$ is a (double) dominating set of $G[H]$, it follows that $S$ is a dominating set of $G$.

Let $x \in S \backslash N_{G}(S)$ and let $q \in V(H)$. Since $C$ is a double dominating set of $G[H]$, there exist distinct $(z, c),(w, p) \in C \cap N_{G[H]}[(x, q)]$. Since $x \notin N_{G}(S)$, it follows that $z=w=x$. Thus, $c, p \in T_{x} \cap N_{H}[q]$. Since $(z, c)$ and $(w, p)$ are distinct, $c \neq p$. Thus, $\left|N_{H}[q] \cap T_{x}\right| \geq 2$. Next, let $q, r \in V(H)$ such that $q \neq r$. Then $(x, q) \neq(x, r)$. Since $C$ is an lds of $G[H]$ and $x \notin N_{G}(S)$, it 
follows that $\left|\left(N_{G[H]}[(x, q)] \cup N_{G[H]}[(x, r)]\right) \cap C\right|=\left|\left(N_{H}[q] \cup N_{H}[r]\right) \cap T_{x}\right| \geq 3$. Therefore, $T_{x}$ is a liar's dominating set of $H$ and shows that (i) holds.

Let $x \in S \cap N_{G}(S)$ with $\left|N_{G}(x) \cap S\right|=1$, say $N_{G}(x) \cap S=\{y\}$. Suppose that $\left|T_{y}\right| \leq 2$. First, suppose that $\left|T_{y}\right|=2$ and suppose further that $T_{x}$ is not an almost dominating set of $H$. Then there exist two distinct vertices $a, b \in V(H) \backslash T_{x}$ such that $a, b \notin N_{H}\left(T_{x}\right)$. Thus, $\mid\left(N_{G[H]}(x, a) \cup\right.$ $\left.N_{G[H]}((x, b))\right) \cap C|=|\{y\} \times T_{y}|=| T_{y} \mid=2$, contrary to our assumption that $C$ is an lds of $G[H]$. Hence, $T_{x}$ is an almost dominating set of $H$, showing that $(b)$ holds.

Next, suppose that $\left|T_{y}\right|=1$. Since $C$ is a double dominating set of $G[H], T_{x}$ is a dominating set of $H$. Let $a \in T_{x}$ and suppose that $\mid e p n_{H}\left(a ; T_{x} \mid \geq 2\right.$, say $l, m \in e p n_{H}\left(a ; T_{x}\right)(l \neq m)$. Then $(x, l),(x, m) \notin C$ and $\left|\left(N_{G[H]}((x, l)) \cup N_{G[H]}((x, m))\right) \cap C\right|=|\{(x, a)\}|+\left|T_{y}\right|=2$, contrary to our assumption that $C$ is an $l d s$ of $G[H]$. Thus, $\left|e p n_{H}\left(a ; T_{x}\right)\right| \leq 1$. Suppose now that $\left|e p n_{H}\left(a ; T_{x}\right)\right|=1$, say $d \in e p n_{H}\left(a ; T_{x}\right)$. Since $C$ is an $l d s$ of $G[H], \mid\left(N_{G[H]}[(x, d)] \cup N_{G[H]}[(x, a)]\right)$ $\cap C|=1+| T_{y}|+| N_{H}(a) \cap T_{x} \mid \geq 3$. This implies that $\left|N_{H}(a) \cap T_{x}\right| \geq 1$, that is, $a \in N_{H}\left(T_{x}\right)$, showing that $T_{x} \in \Omega_{H}$. Therefore, (ii) holds.

Let $x \in S \cap N_{G}(S)$ with $N_{G}(x) \cap S=\{y, z\}$ and $\left|T_{y}\right|=\left|T_{z}\right|=1$. Let $T_{y}=\{r\}$ and $T_{x}=\{s\}$. Suppose that $\left|V(H) \backslash N\left[T_{x}\right]\right| \geq 2$. Then there exist $p, q \in V(H) \backslash T_{x}$ such that $N_{G[H]}((x, p)) \cap C=$ $\{(y, r),(z, s)\}=N_{G[H]}((x, q)) \cap C$. This means that $C$ is not an $l d s$ of $G[H]$, contrary to our assumption. Thus, $T_{x}$ is an almost dominating set of $H$. This shows that (iii) holds.

Finally, let $x \in V(G) \backslash S$ with $\left|N_{G}(x) \cap S\right|=1$, say $N_{G}(x) \cap S=\{y\}$. Let $a, b \in V(H)$, where $a \neq b$. Then $(x, a),(x, b) \notin C$. Since $C$ is liar's dominating set of $G[H]$, there exist at least three distinct vertices $(v, b),(w, c),(z, d) \in C \cap\left(N_{G[H]}((x, a)) \cup N_{G[H]}((x, b))\right)$. This implies that $v=$ $w=z=y$, and $b, c, d \in T_{y}$. Hence, $\left|T_{y}\right| \geq 3$, showing that (a) holds. Suppose now that $N_{G}(x) \cap$ $S=\{y, z\}$. Let $r, s \in V(H)$, where $r \neq s$. Then $(x, r),(x, s) \notin C$. Since $C$ is an lds of $G[H]$, it follows that $\left|\left(N_{G[H]}[(x, r)] \cup N_{G[H]}[(x, s)]\right) \cap C\right|=\left|\{y\} \times T_{y}\right|+\left|\{z\} \times T_{z}\right|=\left|T_{y}\right|+\left|T_{z}\right| \geq 3$. Hence, $\left|T_{y}\right| \geq 2$ or $\left|T_{z}\right| \geq 2$. This shows that (iv) holds.

For the converse, suppose that $S$ is a dominating set of $G$ and satisfies properties (i), (ii), (iii) and (iv). Let $(x, a) \in V(G[H])$ and consider the following cases:

\section{Case 1. $x \notin S$}

Since $S$ is a dominating set of $G$, it follows that $\left|N_{G}(x) \cap S\right| \geq 1$. Clearly, $\left|N_{G[H]}[(x, a)] \cap C\right| \geq$ 2 if $\left|N_{G}(x) \cap S\right| \geq 2$. So suppose that $\left|N_{G}(x) \cap S\right|=1$, say $N_{G}(x) \cap S=\{y\}$. Then, by (iv)(a), $\left|N_{G[H]}[(x, a)] \cap C\right|=\left|T_{y}\right| \geq 3$.

\section{Case 2. $x \in S$}

If $x \in S \backslash N_{G}(S)$, then $T_{x}$ is an $l d s$ of $H$ by $(i)$. Hence, $\left|N_{G[H]}[(x, a)] \cap C\right|=\left|N_{H}[a] \cap T_{x}\right| \geq 2$. Suppose that $x \in S \cap N_{G}(S)$. Suppose first that $\left|N_{G}(x) \cap S\right|=1$. Then by $(a)$, (b), and (c) of ( $i$ i ), 
we have $\left|N_{G[H]}[(x, a)] \cap C\right| \geq 2$. Suppose that $\left|N_{G}(x) \cap S\right|=2$. Then $\left|N_{G[H]}[(x, a)] \cap C\right| \geq 2$ by (iii).

Therefore, $C$ is a double dominating set of $G[H]$.

Next, let $(x, a),(\nu, b) \in V(G[H])$, where $(x, a) \neq(\nu, b)$. If $x \neq v$, then, by (i), (ii), (iii), and (iv), it can be easily shown that $\left|\left(N_{G[H]}[(x, a)] \cup N_{G[H]}[(v, b)]\right) \cap C\right| \geq 3$. Suppose that $x=v$. Then $a \neq b$. Consider the following cases:

Case 1. $x \notin S$

Then, by (iv), $\left|\left(N_{G[H]}[(x, a)] \cup N_{G[H]}[(v, b)]\right) \cap C\right| \geq 3$.

Case 2. $x \in S$

Sub-case 1. $x \notin N_{G}(S)$

Then, by (i), $T_{x}$ is a liar's dominating set of $H$. Hence, $\left|\left(N_{G[H]}[(x, a)] \cup N_{G[H]}[(\nu, b)]\right) \cap C\right|=$ $\left.\mid\left(N_{H}[a] \cup N_{H}[b]\right) \cap T_{x}\right) \mid \geq 3$.

Sub-case 2. $x \in N_{G}(S)$

Then, by (ii) and (iii), $\left|\left(N_{G[H]}[(x, a)] \cup N_{G[H]}[(\nu, b)]\right) \cap C\right| \geq 3$.

Accordingly, $C$ is a liar's dominating set of $G[H]$.

Corollary 4.2. Let $G$ and $H$ be connected graphs of orders $n \geq 2$ and $m \geq 3$, respectively. Then $\gamma_{L R}(G[H]) \leq \min \left\{\gamma(G) \gamma_{L R}(H), 3 \gamma_{t}(G)\right\}$.

Proof. Let $S_{1}$ and $S_{2}$ be $\gamma$-set and $\gamma_{t}$-set of $G$, respectively, and let $D$ be a $\gamma_{L R}$-set of $H$. By Theorem 4.1, $C_{1}=\cup_{x \in S_{1}}\left[\{x\} \times T_{x}\right]$ and $C_{2}=\cup_{y \in S_{2}}\left[\{y\} \times E_{y}\right]$, where $T_{x}=D$ for each $x \in S_{1}$ and $E_{y}=\{a, b, c\} \subseteq V(H)$ for each $y \in S_{2}$, are $l d s$ of $G[H]$. Thus, $\gamma_{L R}(G[H]) \leq \min \left\{\left|C_{1}\right|,\left|C_{2}\right|\right\}=$ $\min \left\{\gamma(G) \gamma_{L R}(H), 3 \gamma_{t}(G)\right\}$.

Remark 4.3. Both the upper bound and the strict inequality in Corollary 4.2 can be attained.

To see this, consider $P_{3}\left[P_{3}\right], P_{4}\left[P_{7}\right]$ in Figure 2, and $G\left[C_{3}\right]$ in Figure 3.

It can be verified that $\gamma_{L R}\left(P_{3}\left[P_{3}\right]\right)=3=\gamma\left(P_{3}\right) \gamma_{L R}\left(P_{3}\right)<6=3 \gamma_{t}\left(P_{3}\right), \gamma_{L R}\left(P_{4}\left[P_{7}\right]\right)=6=3 \gamma_{t}\left(P_{4}\right)$ $<12=\gamma\left(P_{4}\right) \gamma_{L R}\left(P_{7}\right)$, and $\gamma_{L R}\left(G\left[C_{3}\right]\right)=4<6=\min \left\{\gamma(G) \gamma_{L R}\left(C_{3}\right), 3 \gamma_{t}(G)\right\}$. 

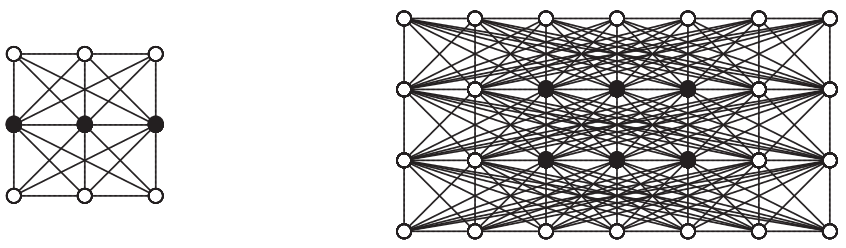

$$
P_{3}\left[P_{3}\right] \quad P_{4}\left[P_{7}\right]
$$

Figure 2: The graphs $P_{3}\left[P_{3}\right]$ and $P_{4}\left[P_{7}\right]$.

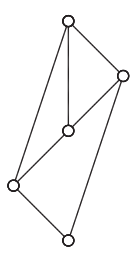

G

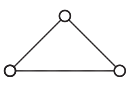

$C_{3}$

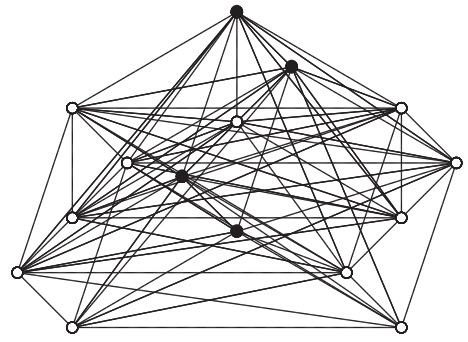

$G\left[C_{3}\right]$

Figure 3: The graphs $G, C_{3}$, and $G\left[C_{3}\right]$.

\section{Acknowledgement}

The authors would like to thank the referee for his or her invaluable comments and suggestions.

\section{References}

[1] T. W. Haynes, S. T. Hedetniemi and P. J. Slater, Fundamentals of Domination in Graphs, Monographs and textbooks in Pure and Applied Mathematics, Marcel Dekker, New York, NY, USA, 208(1998).

[2] M. Nikodem, False alarm in fault-tolerant dominating sets in graphs, Opuscula Mathematica, 32(2012), 751760.

[3] P. J. Slater, Liar's domination, Networks, 54 (2009), 70-74.

[4] P. J. Slater and M. L. Roden, Liar's domination in graphs, Discrete Mathematics, 309(2008), 5884-5890.

Department of Mathematics and Statistics, College of Science and Mathematics, MSU-Iligan Institute of Technology, 9200 Iligan City, Philippines.

E-mail: carlito_balandra@yahoo.com

Department of Mathematics and Statistics, College of Science and Mathematics, MSU-Iligan Institute of Technology, 9200 Iligan City, Philippines.

E-mail: serge_canoy@yahoo.com 